\title{
Nonlinearity and Facilitation in Phosphoinositide Signaling Studied by the Use of Caged Inositol Trisphosphate in Xenopus Oocytes
}

\author{
I. Parker and R. Miledi \\ Laboratory of Cellular and Molecular Neurobiology, Department of Psychobiology, University of California Irvine, California \\ 92717
}

The phosphoinositide signaling pathway, which mediates neurotransmitter responses, was studied in Xenopus oocytes by recording membrane currents evoked using lightflash photolysis of caged inositol trisphosphate (caged $\left.\right|_{3}$ ) to produce rapid and reproducible transients in intracellular $\mathrm{IP}_{3}$ levels. Photolysis of caged $\mathrm{IP}_{3}$ evoked currents which were carried largely by chloride ions and depended upon intracellular, but not extracellular, calcium. A given light flash evoked larger responses when the amount of caged $\mathrm{IP}_{3}$ loaded into the oocyte was increased, and illumination of the vegetal hemisphere gave larger responses than the animal. Long (10 sec) light exposures produced oscillatory currents, resembling responses to serotonin and other agonists, which became larger, more transient, and of shorter latency as the light intensity was increased. Brief (ca. $100 \mathrm{msec}$ ) flashes evoked a single "spike" of current. The caged IP ${ }_{3}$ response showed a threshold, in that light flashes had to be greater than a certain intensity and duration before currents could be detected. Associated with this, sub- and suprathreshold light flashes caused a long-lasting (seconds or minutes) potentiation of responses to subsequent test flashes. The lightflash response was also potentiated by a preceding intracellular injection of $\mathrm{IP}_{3}$ and by extracellular application of an agonist thought to induce $\mathrm{IP}_{3}$ liberation. However, intracellular injections of calcium depressed the response. We conclude that the liberation of calcium from intracellular stores varies nonlinearly with the intracellular level of $\mathrm{IP}_{3}$. This phenomenon may explain earlier observations, including the long latency of currents evoked by low doses of agonists such as acetylcholine and serotonin, and the nonlinear facilitation seen between these agonists. Further, it suggests a mechanism for "chemical integration," which may be important In the functioning of neurons and other cells which use $\mathbf{I P}_{\mathbf{3}}$ as an intracellular messenger.

Oocytes of Xenopus laevis possess a phosphoinositide signaling system by which the activation of muscarinic and other receptors leads to the generation of an oscillatory chloride membrane current (Kusano et al., 1982; Miledi and Parker, 1984; Gundersen et al., 1984; Dascal et al., 1985; Oron et al., 1985; Parker et al., 1985; Parker and Miledi, 1986, 1987b; Gillo et al., 1987; Takahashi et al., 1987). The functioning of this system appears

Received Dec. 12, 1988; revised May 3, 1989; accepted May 5, 1989.

This work was supported by grants GM39831 and NS23284 from the U.S. Public Health Service.

Correspondence should be addressed to Dr. I. Parker at the above address.

Copyright @ 1989 Society for Neuroscience 0270-6474/89/114068-10\$02.00/0 similar to that described in many other cell types, including neurons (Berridge and Irvine, 1984; Hokin, 1985; Berridge, 1986, $1987,1988 \mathrm{~b})$. Bricfly, reccptor binding lcads, via the activation of a GTP-binding protein, to the cleavage of membrane phosphatidylinositol 4,5-bisphosphate by a phosphoinositidase enzyme to yield inositol 1,4,5-trisphosphate $\left(\mathrm{IP}_{3}\right)$ and diacylglycerol. $\mathrm{IP}_{3}$ then acts as an intracellular messenger to release calcium from intracellular stores, and the resulting rise in free calcium in turn activates calcium-dependent chloride channels to give the final membrane current response.

The large size ( $>1 \mathrm{~mm}$ diameter) of the oocyte and the ability to monitor intracellular calcium by recording chloride currents make the oocyte an especially suitable cell in which to study the phosphoinositide signaling pathway. In particular, it is possible to bypass receptor activation and examine the responses obtained when $\mathrm{IP}_{3}$ is directly injected into the oocyte (Oron et al., 1985; Parker and Miledi, 1986, 1987a, b; Miledi et al., 1987; Berridge, 1988a). However, some problems remain with this approach. One is the practical difficulty in achieving repeatable injections of $\mathrm{IP}_{3}$ through micropipettes, which frequently become plugged. A second is that injection is likely to produce an unphysiologically high level of $\mathrm{IP}_{3}$ in a localized region around the pipette tip, unlike the more diffuse release which will occur when agonists are bath-applied to activate receptors over the oocyte surface.

We describe here a study of $\mathrm{IP}_{3}$-activated currents in the oocyte, made using caged $\mathrm{IP}_{3}$ so as to circumvent these difficulties. Caged $\mathrm{IP}_{3}$ is a photolabile derivative of $\mathrm{IP}_{3}$, which is physiologically inactive but, on irradiation with UV light, decomposes to liberate free $\mathrm{IP}_{3}$, together with a proton and 2-nitrosoacetophenone (Nerbonne, 1986; Gurney and Lester, 1987; Walker et al., 1987). Thus, we were able to load caged $\mathrm{IP}_{3}$ into oocytes and allow it, in the dark, to distribute throughout the cell. Appropriate illumination was then used to photolyse a small fraction of the caged $\mathrm{IP}_{3}$, causing the liberation of free $\mathrm{IP}_{3}$ and the subsequent generation of a chloride current. An advantage of this technique was that the intensity and duration of the photolysing light flash could be varied to give a precise control of the amount of $\mathrm{IP}_{3}$ liberated and, because only a small fraction of the caged $\mathrm{IP}_{3}$ was consumed during each flash, many reproducible responses could be recorded from each oocytc.

An abstract describing some of this work has appeared (Parker, 1988).

\section{Materials and Methods}

Voltage-clamp recording from oocytes. Experiments were made on ovarian oocytes of Xenopus laevis obtained following the death of the donor 
frogs. Oocytes were treated with collagenase to remove follicular and other enveloping cells (Miledi and Parker, 1984), and membrane currents were recorded using a 2-electrode voltage clamp (Kusano et al., 1982; Miledi, 1982). The membrane potential was usually held at -60 $\mathrm{mV}$, and during recording the oocytes were continually superfused with Ringer's solution at room temperature $\left(20-24^{\circ} \mathrm{C}\right)$. Records of membrane current were usually low-pass-filtered at $200 \mathrm{~Hz}$. Normal Ringer's solution contained (in $\mathrm{mM}$ ); $\mathrm{NaCl}, 120 ; \mathrm{KCl}, 2 ; \mathrm{CaCl}_{2}, 1.8 ; \mathrm{HEPES}, 5$; at pH about 7.0. Zero calcium Ringer's contained no added calcium, and additionally $2 \mathrm{~mm} \mathrm{MgCl}{ }_{2}$ and $1 \mathrm{mM}$ EGTA. Intracellular injections into the oocyte were made through glass micropipettes, using pneumatic pressure pulses. The volumes of fluid injected were estimated by measuring the diameters of fluid droplets expelled with the pipette tip in air (Parker and Miledi, 1987b). Calcium was injected as a $5 \mathrm{~mm}$ aqueous solution, and EGTA as an $80 \mathrm{~mm}$ solution adjusted to $\mathrm{pH} 7$ with $\mathrm{KOH}$. $\mathrm{IP}_{3}$ (myo-inositol 1,4,5-trisphosphate; Calbiochem, La Jolla) was injected as a $10 \mu \mathrm{m}$ solution, together with $50 \mu \mathrm{M}$ EDTA and $5 \mathrm{~mm}$ HEPES at $\mathbf{p H} 7$.

Caged $\mathrm{IP}_{3}$. Caged $\mathrm{IP}_{3}$ [myo-inositol 1,4,5-trisphosphate, $\mathrm{P}^{4(5)}-1-(2-$ nitrophenyl) ethyl ester] was obtained from Calbiochem (La Jolla) and was specified by the manufacturer to be a mixlure of isomers with a cage present on either the 4 or 5 , but not the 1 position of $\mathrm{IP}_{3}$. It was injected as a $1 \mathrm{~mm}$ aqueous solution together with $50 \mu \mathrm{M}$ EDTA and $5 \mathrm{~mm}$ HEPES at $\mathrm{pH} 7$. The stock solution was stored in the dark at $-20^{\circ} \mathrm{C}$, and care was taken to minimize exposure to light at all times. Each oocyte was loaded with between $0.2-3$ pmol of caged $\operatorname{IP}_{3}(0.2-3 \mathrm{nl}$ of solution). The injection pipette was then withdrawn, and the oocyte was allowed to recover for about $1 \mathrm{hr}$ before beginning recordings. Identical procedures were used in control experiments where oncytes were loaded with caged ATP (Calbiochem).

Photolysis of caged $\mathrm{IP}_{3}$ was achieved by focusing light from a continuously burning $75 \mathrm{~W}$ xenon arc lamp (Photon Technology) onto the oocyte through an epifluorescence microscope (Zeiss) fitted with a $6 \times$ Neofluar objective. The light intensity was altered either by varying the lamp current or by means of a neutral density wedge inserted in the light path. An electromagnetic shutter (constructed from a polygraph pen motor) controlled the duration of light exposure, and this operated with a rise and fall time of about $5 \mathrm{msec}$. For practical convenience, most experiments were done by varying the duration of the light flash incident on the oocyte, while maintaining a constant light intensity. A photomultiplier placed on the microscope phototube monitored the flash duration and gave a relative measure of light intensity. An aperture diaphragm was used to set the area of the light incident on the oocyte. This could be adjusted to cover the whole oocyte or restricted to a spot with a minimum diameter of about $200 \mu \mathrm{m}$, which could be positioned at any desired location. To study the interactions between the responses to photolysis of caged $\mathrm{IP}_{3}$ and responses to intracellular injections of second messengers or local extracellular application of agonist, the light spot was reduced to $200 \mu \mathrm{m}$ diameter and was centered on the tip of the messenger or agonist application pipette. No filters were interposed in the light path, but the incident light was restricted to wavelengths between about 350 and $500 \mathrm{~nm}$, limited at short wavelengths by absorption in the glass lenses and at long wavelengths by the dichroic mirror in the epifluorescence system. A pronounced facilitation was observed between responses to successive light flashes (see below). Intervals of at least $50 \mathrm{sec}$ (usually $100 \mathrm{sec}$ ) were, therefore, allowed before obtaining each record.

\section{Results}

\section{Loading of caged $\mathrm{IP}_{3}$}

Oocytes were impaled with $2 \mathrm{KCl}$-filled microelectrodes, voltage-clamped at $-60 \mathrm{mV}$, and then allowed to settle for a few minutes before insertion of the micropipette containing caged $\mathrm{IP}_{3}$. Each oocyte was loaded with, usually, 0.5-1 pmol of caged $\mathrm{IP}_{3}$, applied with 10-100 pneumatic pressure pulses over a few minutes. Apart from a dim light required to position the pipettes, all procedures were done in near darkness. Nevertheless, injection of the caged $\mathrm{IP}_{3}$ obtained from Calbiochem elicited large (several hundred $\mathrm{nA}$ ) inward oscillatory membrane currents. These responses may have arisen because of contamination by free $\mathrm{IP}_{3}$ or because of the presence of some $\mathrm{IP}_{3}$ caged at the the $P_{1}$ position, which is biologically active (Walker et al., 1987). Whatever the case, the oscillatory currents declined over several minutes following loading, and oocytes were left for an hour or more before recording, at which time the oscillations had ceased entirely and the current trace had returned to the original baseline.

\section{Membrane currents evoked by photolysis of caged $I P_{3}$}

Light flashes applied to more than 20 control oocytes (not loaded with caged $\mathrm{IP}_{3}$ ) gave no or very small ( $<5 \mathrm{nA}$ ) currents coincident with the light flash, which probably arose because of temperature changes (Miledi et al., 1987; Parker, 1989). In contrast, similar or much shorter light flashes applied to oocytes loaded with caged $\mathrm{IP}_{3}$ evoked large inward currents, which began after a latency of between a few hundred msec and a few sec after the onset of illumination (Figs. 1,2). Almost certainly these responses arose because of the liberation of $\mathrm{IP}_{3}$, and not some other product of the photolysis reaction, since they were not seen in 3 oocytes loaded with similar or greater amounts of caged ATP.

It was possible to evoke many (tens or even hundreds) of responses in a single oocyte without depleting the available store of caged $\mathrm{IP}_{3}$. This is not surprising, since oocytes were generally loaded with about $10^{-12} \mathrm{~mol}$ of caged $\mathrm{IP}_{3}$, while currents can be evoked by injection of as little as $10^{-15}-10^{-16} \mathrm{~mol}$ of free $\mathrm{IP}_{3}$ (Parker and Miledi, 1987b). Thus, each light flash probably photolysed only a tiny fraction of the caged $\mathrm{IP}_{3}$ loaded into the oocyte.

Figure 1 illustrates membrane current responses evoked by relatively long $(10 \mathrm{sec})$ exposures to light. At low light levels these responses began after a delay of several sec (Fig. 1, $A, B$ ), showed oscillations which then continued during the light exposure, and subsided within about $10 \mathrm{sec}$ after it was extinguished. As the light intensity was increased, the latency to onset became shorter and the peak size of the current grew. Also, the response became increasingly transient, so that at maximal intensity a "spike" of current lasting only about $2 \mathrm{sec}$ was observed, despite continued illumination. The phasic nature of this response could arise from inactivation of some stage in the signaling pathway or of the chloride channels mediating the current (Boton et al., 1989), but at present we cannot exclude the possibility that the caged $\mathrm{IP}_{3}$ near the surface membrane was consumed, and subsequently replenished between stimuli by diffusion from deeper regions of the oocyte where it was partially shielded by the nearly opaque cytoplasm.

\section{Nonlinear relation between light and evoked current}

Most experiments were done using light flashes with durations of a few hundred msec or less, which were brief in comparison to the duration of the membrane current they evoked. Oocytes usually responded to these stimuli with a single "spike" of current lasting 1 or $2 \sec$ (Fig. 2, $A, C$ ), although oscillations in current were sometimes seen on the falling phase of this response (e.g., second trace in Fig. 2D). The peak size of the current increased when either the intensity (Fig. $2, A, B$ ) or the duration (Fig. 2, $D, E$ ) of the light flash were increased, but a striking feature was that no responses could be detected (less than a few $n A)$ when the intensity or duration were below a certain level. All $(>30)$ oocytes examined showed this threshold phenomenon. As the intensity or duration were increased above threshold, the response grew progressively, and the latency to onset of the current became shorter.

To analyze further the stimulus-response relation for the cur- 


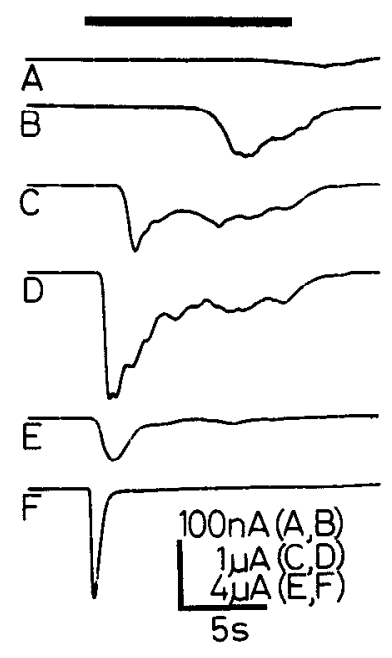

Figure 1. Membrane currents evoked in an oocyte by photolysis of intracellularly loaded caged $\mathrm{IP}_{3}$. Traces show membrane current, recorded at a clamp potential of $-60 \mathrm{mV}$, in response to light of increasing intensities applied for the duration indicated by the bar. The light intensities for each trace, expressed as a percentage of the maximum were as follows: $A, 0.9 ; B, 3 ; C, 11 ; D, 16 ; E, 25 ; F, 100$. In this, and other figures, downward deflections correspond to inward membrane currents. The oocyte was positioned with the vegetal pole facing the light, and the diameter of the light spot was set to cover the whole hemisphere. Notc that the recording gain was reduced as the light intensity was increased.

rent evoked by photolysis of caged $\mathrm{IP}_{3}$, the measurements were plotted on double-logarithmic coordinates. On these coordinates, a dose-response relationship is expected to follow a straight line at low levels of activation and to become less steep and approach a maximal responsc as the stimulus is progressivcly increased. The limiting slope (Hill coefficient) of the linear part of the relationship provides a measure of the cooperativity of the response (see, for example, Colquhoun, 1973). Data are illustrated in Figure 2, $C, F$, from 4 oocytes where response sizes were measured with different intensities or durations of light exposure. The Hill coefficients for the oocytes shown varied between 2.6 and 3.7, and a mean value oblained in 9 oocytes was $3.7 \pm 0.4$ (SEM), pooling experiments where the light duration or intensity were varied. In most experiments, the strongest stimuli employed were not sufficient to saturate the total current that could be evoked. However, the points marked ( $(\bullet)$ in Figure $2 C$ were obtained using a long duration of light, and in this oocyte the current appeared to approach a maximal value of about $6 \mu \mathrm{A}$ as the light intensity was raised.

Both the threshold level of light and the sizes of the responses evoked by suprathreshold flashes depended upon the amount of caged $\mathrm{IP}_{3}$ loaded into the oocyte. For example, one oocyte was first loaded with about $0.3 \mathrm{pmol}$ of caged $\mathrm{IP}_{3}$. After waiting an hour for this to distribute throughout the cell, the threshold flash duration to give a detectable response was $600 \mathrm{msec}$, and a flash duration of $1200 \mathrm{msec}$ elicited a current of $300 \mathrm{nA}$. The oocyte was then loaded with a further 3 pmol caged $\mathrm{IP}_{3}$, and recordings were again made after waiting for about $1 \mathrm{hr}$. After loading the additional caged $\mathrm{IP}_{3}$, the threshold flash duration (at the same intensity) was reduced to $15 \mathrm{msec}$, and a current of $350 \mathrm{nA}$ was evoked by a flash of $40 \mathrm{msec}$ duration.
Different sensitivity to light in animal and vegetal hemispheres

Because the animal hemisphere of the oocyte is pigmented, this might be expected to reduce the transmittance of the photolysis light. We therefore examined whether the sensitivity to light of oocytes loaded with caged $\mathrm{IP}_{3}$ varied in the 2 hemispheres. One approach involved the use of a small ( $250 \mu \mathrm{m}$ diameter) light spot which was positioned close to the equator in either hemisphere. Light flashes with durations $>80 \mathrm{msec}$ evoked currents when the spot was positioned in the vegetal hemisphere, but when it was moved to the animal hemisphere, much longer ( 300 $\mathrm{msec}$ ) flashes were required to give a detectable response. The latencies of the currents evoked in the animal hemisphere were longer than in the vegetal hemisphere, even when comparing responses of similar size (respective mean latencies, $0.50 \pm 0.02$ sec and $0.32 \pm 0.01 \mathrm{sec} ; 7$ trials on each hemisphere, giving currents of 50-200 nA). In this experiment, caged $\mathrm{IP}_{3}$ was loaded through a pipette in the animal hemisphere, so that even if the caged $\mathrm{IP}_{3}$ had not distributed evenly through the oocyte, a higher concentration near the injection site could not explain the lower sensitivity to light in the animal hemisphere. A second approach involved first positioning an oocyte with the animal pole facing the photolysis light, and loading caged $\mathrm{IP}_{3}$ through a pipette in the animal hemisphere. Recordings were made after about $1 \mathrm{hr}$, and the minimum light flash duration required to evoke a response was $1.7 \mathrm{sec}$. The oocyte was then rotated so that the vegetal pole faced the light. Again, a much higher sensitivity was found in the vegetal hemisphere; the threshold flash duration was $0.6 \mathrm{sec}$, and a $1.7 \mathrm{sec}$ flash evoked a current of 600 nA.

Because of the higher observed sensitivity in the vegetal hemisphere, most of the experiments described in this paper were done by illuminating all or a part of this hemisphere.

\section{Current/voltage relationship of the caged $I P_{3}$ response}

The voltage dependence of the current evoked by photolysis of caged $\mathrm{IP}_{3}$ was determined by repeating identical light flashes with the membrane potential clamped to different levels (Fig. $3 A$ ). At $-60 \mathrm{mV}$ the currents were inwardly directed, but as the potential was made progressively less negative, they reduced to zero (Fig. $3 B$ ) and then became outward. A mean value for the reversal of $-23 \mathrm{mV}$ was obtained from 3 oocytes (range, -21 to $-25 \mathrm{mV}$ ), which is in good agreement with that reported for currents evoked by injections of inositol phosphates into the oocyte (Gillo et al., 1987; Parker and Miledi, 1987a) and corresponds to the chloride equilibrium potential in the oocyte (Kusano et al., 1982).

The current/voltage relationship of the caged $\mathrm{IP}_{3}$ response obtained in one oocyte is shown in Figure $3 C$, and similar results were obtained in a further 2 oocytes. A striking feature is that while the current increased steeply as the potential was made more positive than the equilibrium potential, at negative potentials the current increased less and beyond about $-75 \mathrm{mV}$ actually declined, despite the increasing driving force for chloride efflux. This rectification at negative potentials closely resembles the behavior of the chloride currents evoked by intracellular injections of calcium (Miledi and Parker, 1984), and by agonist activation of native acetylcholine $(\mathrm{ACh})$ receptors $(\mathrm{Ku}-$ sano et al., 1982) and induced serotonin (5-HT) receptors (Gundersen et al., 1983). 
A
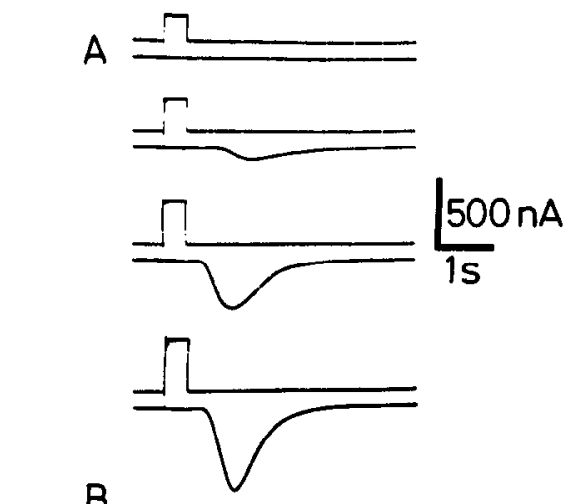

B
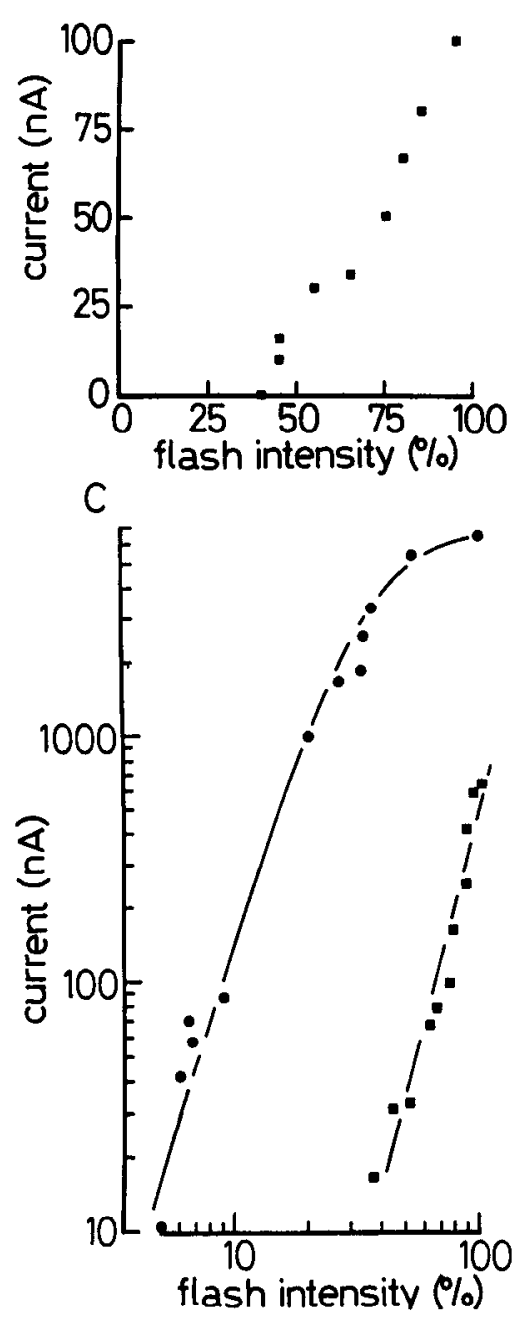

D
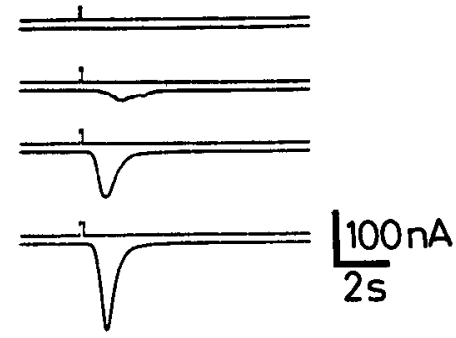

.

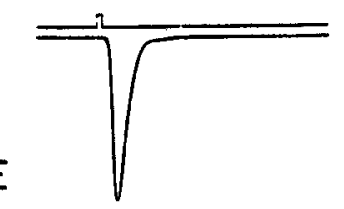

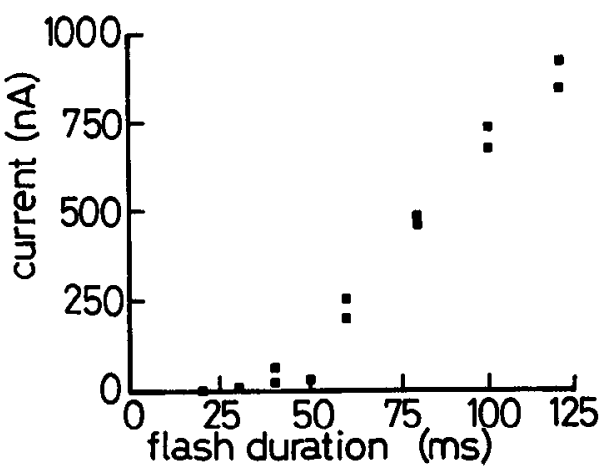

$F$

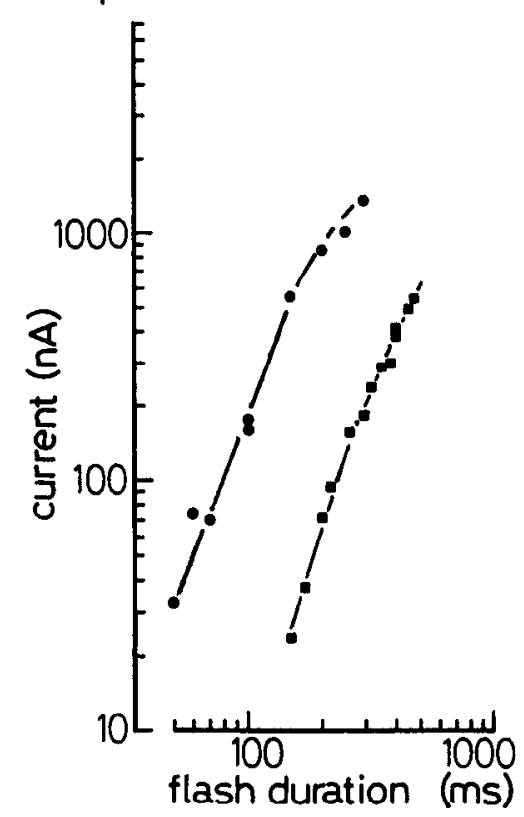

Figure 2. Variation in caged $\mathrm{IP}_{3}$ responses with different intensities (left) or durations (right) of light flash. Experimental records are illustrated in $A$ and $D$, from 2 oocytes which were positioned with their equators facing the light. In each record, the upper trace monitors the light incident on the oocyte, and the lower trace shows membrane current. The intensity of the light was varied in $A$, while maintaining the flash duration constant at $400 \mathrm{msec}$. In $D$. the intensity remained constant, and the flash duration was set to (from top to bottom) $60,80,100,150$, and 180 msec. The upper graphs show measurements of peak sizes of responses plotted on linear scales as a function of flash intensity $(B)$ or flash duration $(E)$. Each graph shows data from single oocytes, which were different from those illustrated above. The lower graphs $(C, F)$ show the variation in response size in a further 4 oocytes, plotted on doublelogarithmic coordinates. Curves are drawn by eye. Data points in $C$ denoted by filled circles were obtained using a light exposure of $12 \mathrm{sec}$ duration, and by filled squares, a $300 \mathrm{msec}$ duration light flash.

\section{Dependence upon intracellular, but not extracellular calcium}

The oscillatory chloride currents evoked by agonists and by intracellular injection of $\mathrm{IP}_{3}$ do not depend upon the presence of calcium in the extracellular solution (Kusano et al., 1977; Dascal et al., 1985; Parker et al., 1985; Parker and Miledi, 1986; Gillo et al., 1987). In agreement with this, the current evoked by photolysis of caged $\mathrm{IP}_{3}$ was not diminished in a solution in which the free calcium was reduced to very low levels (Fig. 4AC).
Although an influx of external calcium is, therefore, unimportant for the generation of the caged $\mathrm{IP}_{3}$ response, it remained possible that the current depends upon intracellular calcium, as do the oscillatory currents activated by agonists and by injections of inositol phosphates (Dascal et al., 1985; Parker et al., 1985; Parker and Miledi, 1987b). To test this, currents were recorded in response to photolysis of caged $\mathrm{IP}_{3}$ while injecting the calcium chelating agent EGTA into the oocyte. Figure $4 D-$ $G$ shows records from an oocyte in which the light spot (250 $\mu \mathrm{m}$ diameter) was positioned on the vegetal hemisphere, a few 


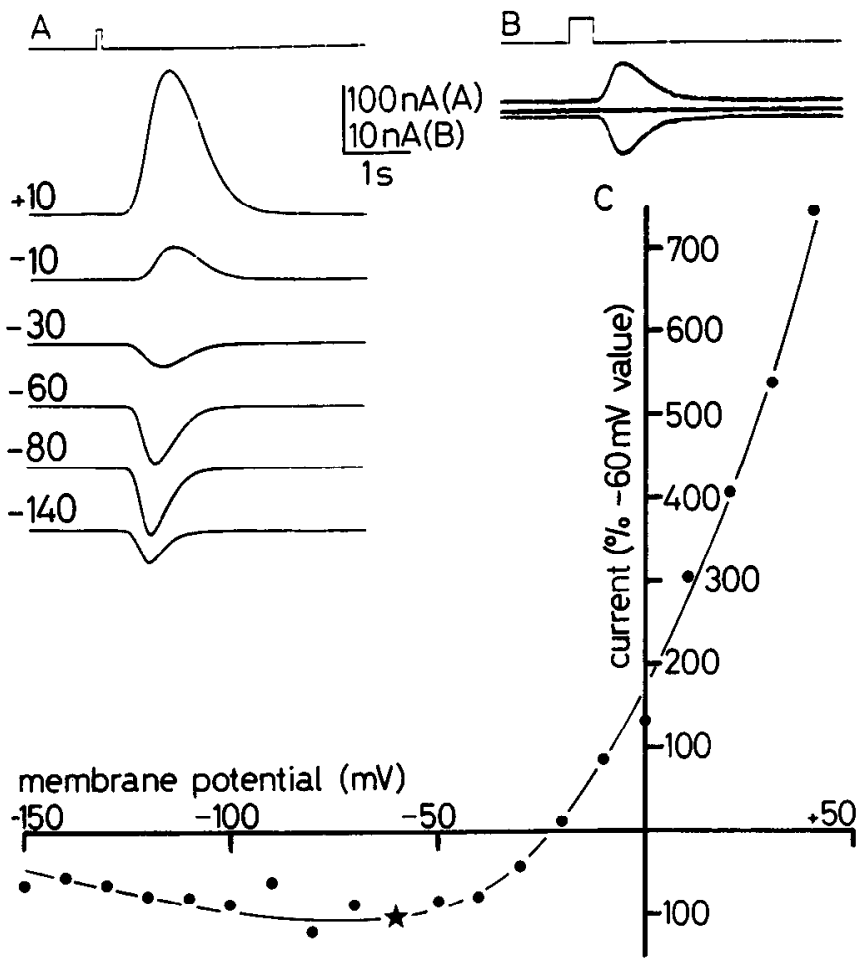

Figure 3. Voltage dependence of the caged $\mathrm{IP}_{3}$ response. $A$, Membrane currents evoked by a constant light flash (upper trace) with the oocyte clamped to different potentials (indicated in $\mathrm{mV}$ ). Light flashes were repeated at an interval of $100 \mathrm{sec}$, and the oocyte was set to the potentials indicated about $90 \mathrm{sec}$ before each record was obtained. The light spot was about $200 \mu \mathrm{m}$ in diameter, positioned close to the equator in the vegetal hemisphere. $B$, Reversal of the caged $\mathrm{IP}_{3}$ response, recorded at a higher gain, and using a longer light flash than in $A$. Traces were obtained at clamp potentials of (from top to bottom) $-22.2,-23.2$, and $-24.2 \mathrm{mV}$. $C$, Current/voltage relation, measured from the same oocyte as in $A$ and $B$. To correct for small changes in sensitivity during the experiment, repeat measurements at $-60 \mathrm{mV}$ were obtained between each trial, and the data points show the peak current at each potential expressed as a percentage of the interpolated value at -60 $\mathrm{mV}$. hundred $\mu \mathrm{m}$ away from the tip of the EGTA-injection pipette inserted in the animal hemisphere. A few minutes after loading as little as 2 pmol EGTA the response to the light flash was reduced to about one-half, and the latency to onset was increased (Fig. $4 E$ ). Injection of an additional 8 pmol EGTA further reduced the response size (Fig. $4 F$ ), and after injecting a total of $30 \mathrm{pmol}$, it was no longer possible to evoke any current, even when the duration of the light pulse was increased from 100 msec to $2 \sec$ (Fig. 4G).

\section{Facilitation by subthreshold light pulses}

As mentioned above, a certain minimum intensity and duration of light flash was required in order to elicit any detectable current. However, although subthreshold flashes did not, by themselves, give any rcsponsc, they did powerfully facilitate the response evoked by a subsequent test flash. For example, Figure 5 shows superimposed traces of currents evoked by a test flash of fixed duration $(220 \mathrm{msec})$ and intensity, which was preceded by conditioning flashes of the same intensities and varying durations. By themselves, neither the test pulse nor any of the conditioning pulses gave a response. However, large currents were obtained when the test flash was preceded by a conditioning flash. These responses became progressively larger and of shorter latency to onset as the duration of the conditioning flash was increased. Marked facilitation with paired light flashes, like that illustrated, was consistently seen in all $(>20)$ oocytes examined.

The facilitatory action of a light flash persisted for many seconds. To measure the time course of decay of the facilitation, superimposed records were obtained in which a fixed conditioning flash was applied near the beginning of each sweep, and test flashes were then given at various times afterwards (Fig. 6). Because of the long-lasting facilitation, intervals of $100 \mathrm{sec}$ were allowed for recovery between each pair of flashes. In the experiment illustrated, the conditioning and test flashes were of the same intensity and duration within each frame, but the flash duration was increased between each frame. When the light was brief (Fig. 6A), the size of the response to the test flash declined rapidly with increasing intervals between the conditioning and test flashes, falling to about one-half within $1 \mathrm{sec}$. However, the
Figure 4. Dependence of the caged $\mathrm{IP}_{3}$ response upon intracellular but not extracellular calcium. In all frames, the upper trace is the light monitor, and the lower trace shows clamp current. $A-C$, Effect of calcium-free Ringer's solution. A control response to the light flash is shown in $A$, with the oocyte bathed in normal (1.8 $\mathrm{mm} \mathrm{Ca}{ }^{2+}$ ) Ringer's. The bathing solution was then switched to calcium-free Ringer's, and the four superimposed traces in $(B)$ show responses elicited at $50 \mathrm{sec}$ intervals, beginning $2 \mathrm{~min}$ after the solution change. Finally, the record in $C$ was obtained about $5 \mathrm{~min}$ after returning to normal Ringer's. $D-G$, Abolition of the caged $\mathrm{IP}_{3}$ response by intracellular loading with EGTA. Records show a control response $(D)$ and responses evoked after loading the oocyte with a total of 2 $(E), 10(F)$, and $30(G)$ pmol of EGTA.
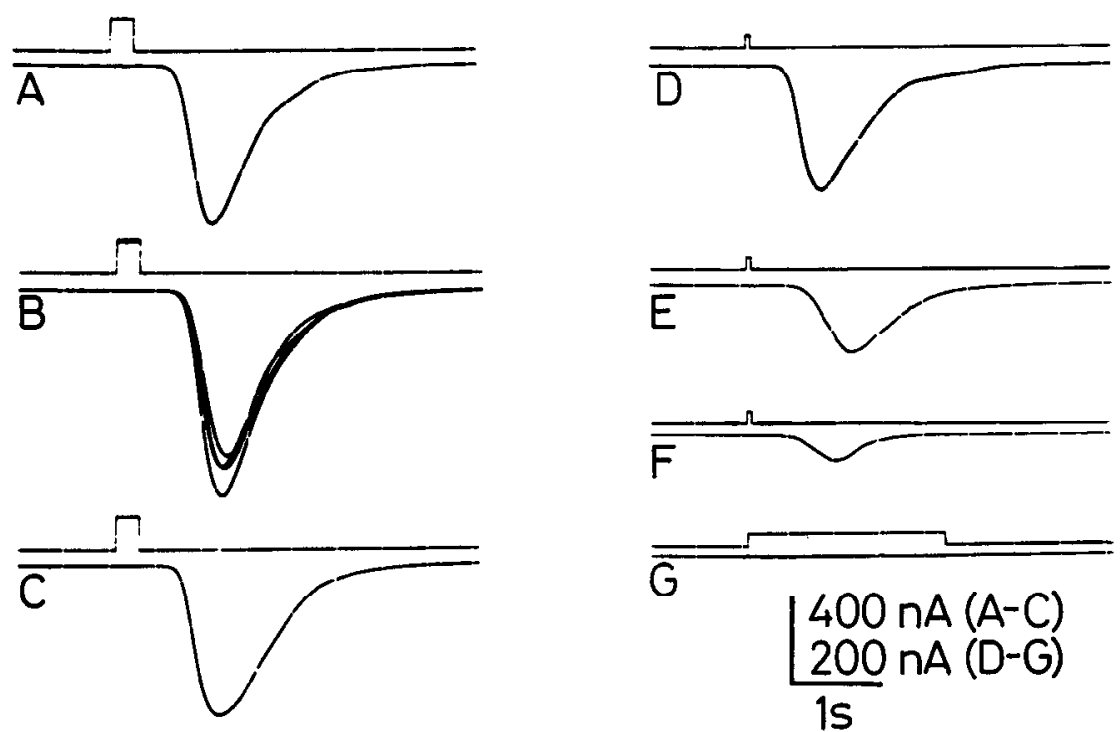


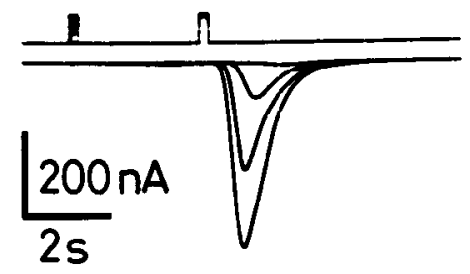

Figure 5. Facilitation of the caged $\mathrm{IP}_{3}$ response by conditioning light flashes. Upper trace is light monitor, lower trace is clamp current. Four superimposed records show currents evoked by a test light flash of constant $(220 \mathrm{msec})$ duration, which was preceded by conditioning flashes of the same intensities and durations of 50,100, 150, and $200 \mathrm{msec}$. The light illuminated the whole oocyte, which was positioned with the equator facing the illuminator. Intervals of $100 \mathrm{sec}$ were allowed between each pair of stimuli.

decline of facilitation became slower as the flash duration was increased, and with a just suprathreshold flash (Fig. 6D), the half-time of decay was about $10 \mathrm{sec}$. In addition to the decline in response size with increasing interval between light flashes, it is also evident in Figure 6 that the latency to onset of the current became longer.

\section{Interaction between responses to caged and free $\mathrm{IP}_{3}$}

The facilitation seen with conditioning light flashes might arise if a threshold amount of $\mathrm{IP}_{3}$ is required to evoke a detectable current, so that the subthreshold level of $\mathrm{IP}_{3}$ released by a conditioning flash summates with that released by the test flash to produce a level sufficient to give a membrane current. However, a less attractivc possibility is that a subthreshold light may fail to release any free $\mathrm{IP}_{3}$, but somehow weakens the "cage," so that a subsequent flash is able to liberate more $\mathrm{IP}_{3}$. There does not appear to be any indication that caged $\mathrm{IP}_{3}$ behaves in this way (Gurney and Lester, 1987; Walker et al., 1987), but to further discount the possibility, we looked for facilitation between responses evoked by photolysis of caged $\mathrm{IP}_{3}$ and intracellular injection of free $\mathrm{IP}_{3}$. Facilitation would be expected if the idea of a threshold level of $\mathrm{IP}_{3}$ is correct, but not if a conditioning light were serving only to weaken the bond "caging" the $\mathrm{IP}_{3}$.

An experiment to test this point is shown in Figure 7. An oocyte which had been loaded with caged $\mathrm{IP}_{3}$ was penetrated by an additional pipette containing free $\mathrm{IP}_{3}$, and the photolysis light was focused as a $200 \mu \mathrm{m}$ spot centered on the site of impalement. The flash duration was set so that small $(5 \mathrm{nA})$ currents were evoked by flashes repeated at $90 \mathrm{sec}$ intervals. After obtaining several control responses to the light flash, an injection of $\mathrm{IP}_{3}$ was given $40 \mathrm{sec}$ before a flash. This injection elicited only a small ( $15 \mathrm{nA})$ oscillatory current, but the response to the flash was greatly potentiated (Fig. $7 C$ ), and potentiation was still evident to a subsequent flash $90 \mathrm{sec}$ later (Fig. $7 D$ ). Results like those illustrated were also obtained in 2 other oocytes.

\section{Facilitation by agonist activation}

Several agonists, including a component of serum (Miledi and Parker, 1989; G. Tigyi, C. M. Matute, and R. Miledi, unpublished observations), generate oscillatory chloride currents in the oocyte and are thought to operate through activation of the phosphoinositide signaling pathway. If this is the case, the response to photolysis of caged $\mathrm{IP}_{3}$ should be facilitated by prior
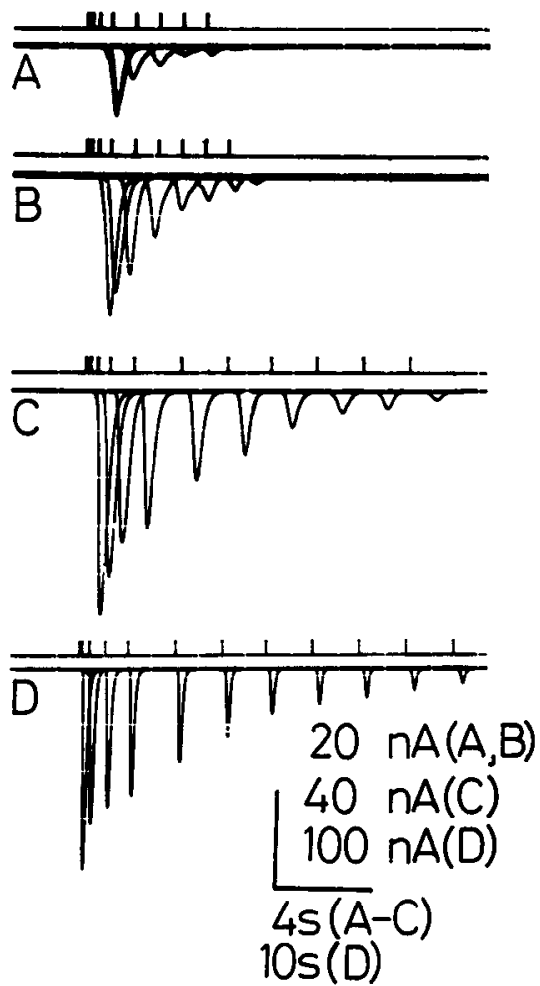

Figure 6. Time course of decay of facilitation following subthreshold conditioning stimuli. Each frame shows superimposed records of currents evoked by test light flashes given at different times after an initial conditioning flash (marked by arrowheads). The durations of the conditioning and test flashes were the same within each frame, and were $55(A), 65(B), 80(C)$, and $150(D) \mathrm{msec}$. The light intensity was constant throughout. Note the changes in recording gain and time base between frames. An interval of $100 \mathrm{sec}$ was allowed between each pair of stimuli.

agonist activation, similar to the facilitation described above with intracellular injection of $\mathrm{IP}_{3}$.

This is demonstrated in Figure 8. A "puff" of serum applied from an extracellular pipette to the oocyte greatly potentiated the response to a just suprathreshold light flash given $30 \mathrm{sec}$ later. Interestingly, facilitation of the caged $\mathrm{IP}_{3}$ response was seen only when the interval between serum application and the light flash was $20 \mathrm{sec}$ or longer, and at intervals shorter than 10 sec the caged $\mathrm{IP}_{3}$ response was not larger than the control (Fig. $8, D, E)$. This observation is consistent with our previous conclusion (Miledi and Parker, 1989) that the liberation of $\mathrm{IP}_{3}$ begins only after a latency of several sec following agonist activation. On the other hand, a light flash given a few seconds before or after a "puff" of serum shortened the latency to onset of the serum response but did not appreciably alter its size. For example, in the oocyte illustrated the latency decreased from $24.5 \pm 1.1$ to $11.3 \pm 0.6 \mathrm{sec}$ (SEM, 8 paired trials), but the peak size changed only slightly from $3.5 \pm 0.4$ to $3.7 \pm 0.2 \mathrm{nA}$.

Interactions between intracellular calcium and caged $I P_{3}$ response

The results described above indicate that a certain threshold amount of $\mathrm{IP}_{3}$ is required to give a detectable membrane current response. This might arise because a certain amount of $\mathrm{IP}_{3}$ is required to cause significant mobilization of intracellular calcium, or because the intracellular free calcium level must rise above a certain value before the membrane chloride channels 


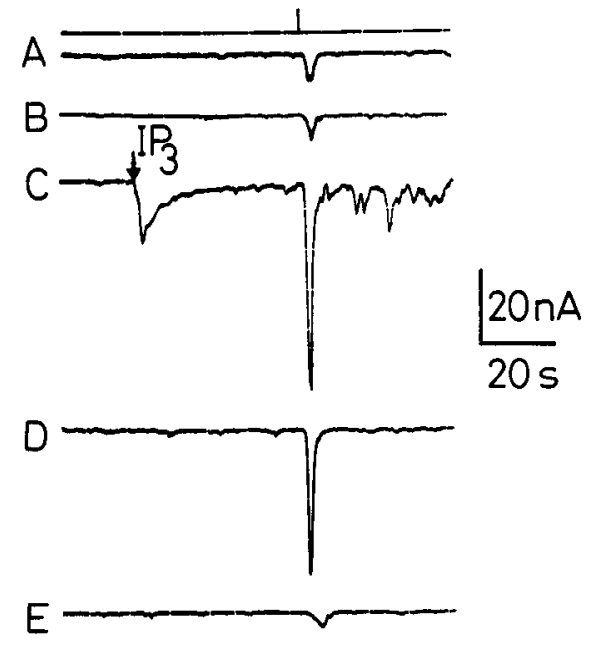

Figure 7. Facilitation of the response to photolysis of caged $\mathbf{I P}_{3}$ by microinjection of free $\mathrm{IP}_{3}$ into the oncyte. Light was focused as a spot of about $200 \mu \mathrm{m}$ diameter, centered on the site of impalement of the $\mathrm{IP}_{3}$ injection pipette in the vegetal hemisphere of the oocyte. Recordings of membrane current $(A-E)$ were obtained at $90 \mathrm{sec}$ intervals in response to identical light flashes (upper trace). $A$ and $B$, Control responses to the light flash. $C$, A single injection of $\mathrm{IP}_{3}$ was made at the arrow, from a pipette containing $10 \mu \mathrm{M} \mathrm{IP}_{3} . D$ and $E$, Responses to light flashes obtained, respectively, 90 and $180 \mathrm{sec}$ after $C$.

become activated. To distinguish between these possibilities, we looked for interactions between the responses to photolysis of caged $\mathrm{IP}_{3}$ and to intracellular injection of calcium.

Injection of calcium into the oocyte evokes a smooth chloride current (Miledi and Parker, 1984). When a light flash was given during or shortly after a response to calcium, the caged $\mathrm{IP}_{3}$ response was depressed (Fig. $9 B$ ), even though a large facilitation could be seen with paired light flashes applied to the same oocyte (Fig. 9E). Interactions between injected calcium and caged $\mathrm{IP}_{3}$ responses were examined in 2 oocytes, using a wide range of calcium injections between those giving no detectable response and responses of a few hundred nA. Potentiation of the caged $\mathrm{IP}_{3}$ response was never observed. Instead, it was usually depressed, except that the response size was not altered by calcium injections giving only small currents.

The lack of facilitation by calcium was further confirmed in another experiment in which calcium or $\mathrm{IP}_{3}$ was injected through a single pipette, filled with an aqueous solution including $5 \mathrm{~mm}$ $\mathrm{CaCl}_{2}$ and $100 \mu \mathrm{M} \mathrm{IP}_{3}$. Either calcium or $\mathrm{IP}_{3}$ could be ejected by applying, respectively, pipette positive or negative ionophoretic currents. Caged $\mathrm{IP}_{3}$ responses evoked by a light flash given $8 \mathrm{sec}$ after an ionophoretic pulse were depressed to $54 \%$ ( $\pm 5 \%$ SEM, 4 trials) of the control value when calcium was injected but were potentiated to $790 \%( \pm 220 \%, 4$ trials $)$ when $\mathrm{IP}_{3}$ was injected.

Figure $9 D$ further illustrates the depressant action of an intracellular calcium injection on the caged $\mathrm{IP}_{3}$ responses evoked by a train of light flashes at $10 \mathrm{sec}$ intervals. Following the calcium pulse, the responses were completely suppressed for about $30 \mathrm{sec}$.

\section{Discussion}

Membrane currents evoked by photolysis of caged IP The membrane currents evoked by light flash photolysis of caged $\mathrm{IP}_{3}$ loaded into oocytes show many similarities with the currents

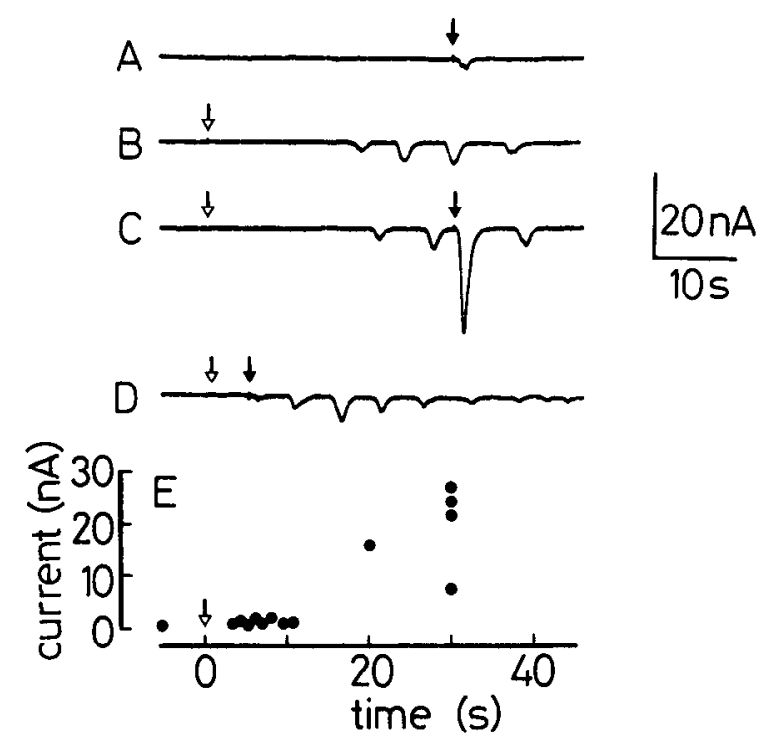

Figure 8. Interaction between responses evoked by extracellular application of agonist and by photolysis of intracellular caged $\mathrm{IP}_{3}$. All records are from a single oocyte. An extracellular pipette containing serum ( $1 / 10$ dilution in Ringer's) was positioned close to the vegetal hemisphere of the oocyte, and the light spot $(200 \mu \mathrm{m}$ diameter) was centered on the tip of this. "Puffs" of serum were ejected by pneumatic pressure pulses as indicated by the open arrows, and flashes of light given as indicated by the filled arrows. Intervals of $90 \mathrm{sec}$ were allowed between each record. $A$ and $B$, Control response to, respectively, the light flash and serum applied separately. $C$ and $D$, Currents evoked when the light flash was given at different times following a puff of serum. $E$, Size of the response to the light flash evoked at different times before and after a puff of serum. Fach point represents a single measurement from records like those in $C$ and $D$. Nominally identical puffs of serum were applied in each record at time zero.

evoked by intracellular injections of free $\mathrm{IP}_{3}$ (Oron et al., 1985; Parker and Miledi, 1986, 1987a, b), and with the currents evoked by agonists, including $\mathrm{ACh}$ and 5-HT (Kusano et al., 1982; Parker et al., 1985; Gillo et al., 1987). Specifically, all of these responses arise largely due to a flux of chloride ions, and while they are independent of extracellular calcium, they are abolished by intracellular loading with the calcium chelating agent EGTA. Thus, the caged $\mathrm{IP}_{3}$ responses almost certainly arise because the light flash liberates free $\mathrm{IP}_{3}$, which subsequently causes the liberation of calcium from intracellular stores, leading finally to the opening of calcium-activated chloride membrane channels.

We did not attempt to quantify how much $\mathrm{IP}_{3}$ would have been liberated by a given light flash (see Walker et al., 1987) because of difficulties in estimating the light which would actually reach the caged $\mathrm{IP}_{3}$ in the oocyte. The cytoplasm of the oocyte absorbs and scatters light strongly, and at wavelengths between 350 and $400 \mathrm{~nm}$, the optical density of a 1/1000 dilution of cytoplasm was measured as about 1.1. Thus, the photolysis light would be attenuated to one-tenth after passage through about $10 \mu \mathrm{m}$ of cytoplasm, indicating that $\mathrm{IP}_{3}$ would be liberated only in a shallow zone within a few $\mu \mathrm{m}$ of the surface membrane.

Although the mechanisms underlying the currents evoked by injection of free $\mathrm{IP}_{3}$ and photolysis of caged $\mathrm{IP}_{3}$ are probably the same, there are marked differences in the time courses of these 2 responses. A pulse of $\mathrm{IP}_{3}$, injected within a few tens of msec, usually gives rise to an initial "spike" of current lasting a few seconds, followed by a slowly decaying current with su- 


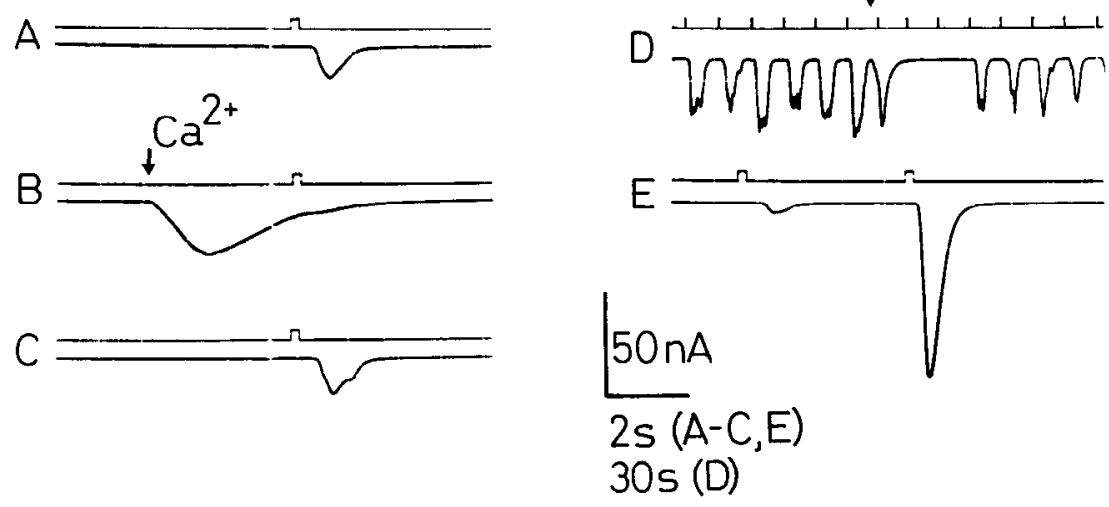

Figure 9. Intracellular injections of calcium do not facilitate the caged $\mathrm{IP}_{3}$ response. Records are from a single oocyte, which was impaled by a $\mathrm{CaCl}_{2}$-filled micropipette in the vegetal hemisphere. The photolysis light was focused as a $200 \mu \mathrm{m}$ spot, centered on the tip of the $\mathrm{Ca}^{2+}$ pipette. In each frame, the upper trace is the light monitor, and the lower shows membrane current. $A-C$, Successive records obtained at 90 sec intervals showing currents evoked by identical light flashes. In $B$, the light was preceded by an injection of $\mathrm{Ca}^{2+}$ (ca. 1 pmol), as indicated by the arrow. $D$, Record at a slower sweep speed, showing inhibition of responses to a train of light flashes at intervals of 10 sec, produced by a single injection of $\mathrm{Ca}^{2+}$ at the time marked by the arrow. E. Facilitation of the response to photolysis of caged $\mathrm{IP}_{3}$ by paired light flashes. The intensity and duration of each flash was the same as in $A-D$.

perimposed oscillations which persist for many minutes (Oron et al., 1985; Parker and Miledi, 1986, 1987b; Gillo et al., 1987). In contrast, a brief (tens or hundreds of msec) light flash applied to an oocyte loaded with caged $\mathrm{IP}_{3}$ generally evokes only a single spike of current, lasting a few seconds. The difference between these responses may arise from the different spatial localization of $\mathrm{IP}_{3}$. Injection of $\mathrm{IP}_{3}$ will create a localized region of high concentration near the tip of the injection pipette. The time course of the current response is, therefore, likely to be influenced by the spread of $\mathrm{IP}_{3}$ from this point source to reach an increasingly large area of surface membrane. Furthermore, the local concentration of $\mathrm{IP}_{3}$ near the pipette tip may be unphysiologically high, and perhaps sufficient to deplete calcium release or saturate the enzymes responsible for the metabolism of $\mathrm{IP}_{3}$. In contrast, the photolysis of caged $\mathrm{IP}_{3}$ is expected to liberate $\mathrm{IP}_{3}$ diffusely throughout a shallow zone of cytoplasm close to the surface membrane, in a manner more closely approximating the release of $\mathrm{IP}_{3}$ in response to agonist activation. Thus, the brief duration of the caged $\mathrm{IP}_{3}$ response probably gives a better indication of the duration of action of $\mathrm{IP}_{3}$ than does the prolonged response to $\mathrm{IP}_{3}$ injection, which may be slowed by the diffusion of $\mathrm{IP}_{3}$ from a point source at some distance from the surface membrane. In agreement, Gillo et al. (1987) found that the nature of the response evoked by injection of $\mathrm{IP}_{3}$ into the oocyte varied with the depth of impalement of the injection pipette. When the pipette tip was inserted deeper into the oocyte, the initial spike of current became smaller, while the subsequent slow oscillatory response was sometimes potentiated and prolonged.

On the other hand, responses evoked by bath application of agonists which activate phosphoinositide signaling in the oocyte show time courses similar to those evoked by prolonged photolysis of caged $\mathrm{IP}_{3}$ (Kusano et al., 1982; Parker et al., 1987; Miledi and Parker, 1989). In both cases, low levels of activation give rise to maintained oscillatory currents, beginning after long delays. As the activation is increased, the currents become larger and begin after shorter latencies. Also, the responses become increasingly transient and, at high agonist concentrations or with intense photolysis light, it is common to see only a brief "spike" of current. Thus, it seems that the rapid decline of current during bath application of high concentrations of agonist may be due in part to the properties of the messenger pathway, rather than because of desensitization of the receptor.

Intracellular injections of calcium (Miledi and Parker, 1984) and inositol phosphates (Berridge, 1988a; I. Parker, unpublished observations) produce larger chloride currents when made into the animal, rather than the vegetal hemisphere of the oocyte. From this, the response to photolysis of caged $\mathrm{IP}_{3}$ would be expected to be greater in the animal hemisphere than in the vegetal; but the opposite sensitivity to light is in fact observed. This discrepancy is probably due to the presence of a layer of pigment lying just under the surface membrane in the animal hemisphere, which would greatly attenuate the light reaching the caged $\mathrm{IP}_{3}$. The present experiments were done, therefore, by illuminating the vegetal hemisphere. We do not know whether the $\mathrm{IP}_{3}$, which is liberated, remains entirely localized to this hemisphere, but it seems that diffusion of $\mathrm{IP}_{3}$ towards the more sensitive animal pole cannot contribute appreciably to the response, since the latency of the current is not shorter when light is focused on the animal hemisphere. Furthermore, responses with indistinguishable latency are evoked by injections of $\mathrm{IP}_{3}$ into the animal and vegetal poles (Berridge, 1988a, b), and focal application of serum to the vegetal pole gives currents with shorter latency than application to the animal pole (Miledi and Parker, 1989).

\section{Nonlinearity of caged $I P_{3}$ responses}

An important finding is that a threshold amount of light is required in order to evoke a detectable response in caged $\mathrm{IP}_{3^{-}}$ loaded oocytes. This might arise if the photolysis of caged $\mathrm{IP}_{3}$ is nonlinear, so that a certain amount of light is required before any $\mathrm{IP}_{3}$ is liberated. However, this is unlikely (Nerbonne, 1986; Gurney and Lester, 1987; Walker et al., 1987) and is not consistent with the finding that responses to near-threshold light flashes can be greatly potentiated by a prior injection of $\mathrm{IP}_{3}$ or by application of an agonist which raises $\mathrm{IP}_{3}$ levels. Instead, it 
seems that the response of the oocyte must vary nonlinearly with the concentration of intracellular $\mathrm{IP}_{3}$, and this conclusion is supported by earlier findings of an apparent threshold for currents evoked by injections of low doses of $\mathrm{IP}_{3}$ (Parker and Miledi, 1987b) and by bath application of low concentrations of agonists (Gundersen et al., 1983; Parker et al., 1987). Two observations indicate that the calcium-activated chloride channels are not responsible for the nonlinearity. First, potentiation of the caged $\mathrm{IP}_{3}$ response was not seen when calcium was injected into the oocyte. Second, the chloride current evoked by calcium injections varies linearly with the dose of calcium (I. Ivorra and I. Parker, unpublished observations). Thus, the nonlinearity probably arises at the stage of calcium liberation by $\mathrm{IP}_{3}$.

Measurements of the currents evoked by different intensities or durations of light flashes (Fig. $2 C, F$ ) demonstrate that, at low levels of activation, the response increases as the 3.7 power of the stimulus magnitude. As discussed above, this power relationship probably reflects the dependence of calcium liberation upon concentration of $\mathrm{IP}_{3}$ and suggests that the opening of intracellular calcium release channels in the oocyte may, therefore, require the cooperative binding of 3 or more molecules of $\mathrm{IP}_{3}$. This result is in good agreement with previous findings of a third-power relationship for $\mathrm{IP}_{3}$-mediated calcium liberation in permeabilized basophilic leukemia cells (Meyer et al., 1988). The steep power function between $\mathrm{IP}_{3}$ level and calcium liberation may explain the apparent threshold in $\mathrm{IP}_{3}$ action, because the amount of calcium liberated will fall sharply as the stimulus is reduced, and the resulting chloride currents could become too small to be detected. However, even if calcium liberation is continuously graded with $\mathrm{IP}_{3}$ level, it remains possible that some other aspect of the signaling pathway (e.g., calcium buffering or uptake) introduces a threshold below which no current is activated. With strong light stimuli, the evoked current appeared to reach a maximal value of about $6 \mu \mathrm{A}$ (Fig. $2 C$ ). Since larger responses may be elicited by injections of calcium into oocytes (Miledi and Parker, 1984), it seems that the saturation of the caged $\mathrm{IP}_{3}$ response does not arise because of maximal activation of membrane chloride channels. Instead, it might be due to a saturation of $\mathrm{IP}_{3}$-mediated calcium release or to a consumption of caged $\mathrm{IP}_{3}$ close to the membrane.

\section{Facilitation and depression}

Responses to photolysis of caged $\mathrm{IP}_{3}$ are potentiated for many seconds following a conditioning light flash. An explanation for this may be found by analogy with the "residual calcium" hypothesis proposed to account for the facilitation of transmitter release at nerve terminals (Katz and Miledi, 1968). Thus, $\mathbf{I P}_{3}$ liberated by the conditioning flash summates with that produced by the test flashes and, because of the nonlinear dose-effectiveness of $\mathrm{IP}_{3}$, gives rise to a much larger response beginning after a shorter latency. In this model, the decay of facilitation is determined by the rate of disappearance of $\mathrm{IP}_{3}$ in the cytoplasm, while the membrane current evoked by a light flash declines more rapidly because of the power-law relationship.

The nonlinearity of $\mathrm{IP}_{3}$ action may account for several characteristics of the phosphoinositide-mediated responses in the oocyte. One of these is the long (many seconds or minutes) latency to onset of membrane currents evoked by low doses of agonists, which has been proposed to arise because the level of some intermediate in the signaling pathway builds up slowly and exceeds a threshold amount required for channel activation only after some time (Kusano et al., 1977, 1982; Parker and
Miledi, 1987b; Berridge et al., 1988). The results presented here demonstrate that such a threshold occurs at the stage of $\mathrm{IP}_{3}$ mediated release of calcium, and we have shown that this can account for much, though not all, of the latency of agonist evoked responses (Miledi and Parker, 1989). A second characteristic is the potentiation seen between responses to agonists such as $\mathrm{ACh}$ and 5-HT. For example, we found (Parker et al., 1987; Miledi et al., 1989) that a dose of 5-HT, which by itself failed to give any response, would still potentiate the response to a low dose of ACh. This may be explained if the levels of $\mathrm{IP}_{3}$ resulting from activation of each receptor summate and, because of the nonlinear relation, evoke a much larger current; although it remains possible that signals from the 2 receptor systems interact also at other stages in the messenger pathway.

An interesting observation is that caged $\mathrm{IP}_{3}$ responses are depressed by a prior injection of calcium into the oocyte. This effect does not appear to arise through desensitization of the calcium-activated chloride channels, since depression was not observed with repeated injections of calcium giving rise to responses of similar size to those used here (Miledi and Parker, 1984). Instead, the depression may be related to the recently reported inhibition by calcium of $\mathrm{IP}_{3}$ binding to its intracellular receptor (Danoff et al., 1988). This negative feedback of calcium upon $\mathrm{IP}_{3}$-mediated calcium liberation could provide an attractive explanation for the origin of the oscillations in chloride current evoked by $\mathrm{IP}_{3}$ (Miledi et al., 1987; Berridge et al., 1988; Payne et al., 1988; Rink and Jacob, 1989).

\section{Relevance to synaptic function}

The mechanisms of phosphoinositide signaling appear to be very similar in many different cell types (Bcrridge, 1986, 1987, 1988 b). In particular, it seems that results obtained in the oocyte should be applicable to the study of neuronal functioning. For example, glutamate stimulates phosphoinositide turnover in neurons (Sladeczek et al., 1985), and, when expressed in the oocyte by injection of brain messenger RNA, glutamate receptors are able to "link in" to the phosphoinositide signaling pathway already present in the native oocyte (Parker et al., 1987; Oosawa and Yamagishi, 1989). Hence, it may be profitable to consider the implications of the present results for the functioning of neurons which use $\mathrm{IP}_{3}$ as a postsynaptic intracellular messenger to mediate slow synaptic responses.

The finding of a threshold level of $\mathrm{IP}_{3}$ required for calcium liberation suggests a basis for a novel, chemical, form of synaptic integration, in which the cellular response depends upon the summation of intracellular $\mathrm{IP}_{3}$ levels. Because of its chemical basis, this mechanism of integration is expected to show properties very different from the electrical integration of synaptic currents. One important characteristic is that facilitation may result from inputs which, by themselves, fail to evoke any detectable electrical signal in the postsynaptic cell. Second, because the decay of facilitation is determined by inositol phosphate metabolism, facilitation is likely to be much longer lasting than the decay times of postsynaptic potentials, which are determined by the membrane time constant. Conversely, the spatial area over which integration occurs will be limited by the intracellular diffusion of $\mathrm{IP}_{3}$ and is thus probably more restricted than for the spread of electrical signals.

\section{References}

Berridge, M. J. (1986) Regulation of ion channels by inositol trisphosphate and diacylglycerol. J. Exp. Biol. 124: 323-335. 
Berridge, M. J. (1987) Inositol trisphosphate and diacylglycerol: Two interacting second messengers. Annu. Rev. Biochem. 56: 159-193.

Berridge, M. J. (1988a) Inositol trisphosphate-induced membrane potential oscillations in Xenopus oocytes. J. Physiol. (Lond.) 403: 589 600.

Berridge, M. J. (1988b) Inositol lipids and calcium signalling. Proc. R. Soc. London [Biol.] 234: 359-378.

Berridge, M. J., and R. F. Irvine (1984) Inositol trisphosphate, a novel second messenger in cellular signal transduction. Nature 312:315321.

Berridge, M. J., P. H. Cobbold, and K. S. R. Cuthbertson (1988) Spatial and temporal aspects of cell signalling. Phil. Trans. R. Soc. London [Biol.] 320: 325-343.

Boton, R., N. Dascal, B. Gillo, and Y. Lass (1989) Two calciumactivated chloride conductances in Xenopus laevis oocytes permeabilized with the ionophore A23187. J. Physiol. (Lond.) 408: 511-534.

Colquhoun, D. (1973) The relation between classical and cooperative models for drug action. In Drug Receptors, H. P. Rang, ed., pp. 149182, Macmillan, London.

Danoff, S. K., S. Supattapone, and S. H. Snyder (1988) Characterization of a membrane protein from brain mediating the inhibition of inositol 1,4,5-trisphosphate receptor binding by calcium. Biochem. J. 254: 701-705.

Dascal, N., B. Gillo, and Y. Lass (1985) Role of calcium mobilization in mediation of acetylcholine-evoked chloride currents in Xenopus laevis oocytes. J. Physiol. (Lond.) 366: 299-314.

Gillo, B., Y. Lass, E. Nadler, and Y. Oron (1987) The involvement of inositol 1,4,5-trisphosphate and calcium in the two-component response to acetylcholine in Xenopus oocytes. J. Physiol. (Lond.) 392: $349-361$.

Gundersen, C. B., R. Miledi, and I. Parker (1983) Serotonin receptors induced by exogenous messenger RNA in Xenopus oocytes. Proc. R. Soc. London [Biol.] 219: 103-109.

Gundersen, C. B., R. Milcdi, and I. Parker (1984) Glutamate and kainate receptors induced by rat brain messenger RNA in Xenopus oocytes. Proc. R. Soc. London [Biol.] 221: 127-143.

Gurney, A. M., and H. A. Lester (1987) Light-flash physiology with synthetic photosensitive compounds. Physiol. Rev. 67: 583-617.

Hokin, L. E. (1985) Receptors and phosphoinositide-generated second messengers. Annu. Rev. Biochem. 54: 205-235.

Katz, B., and R. Miledi (1968) The role of calcium in neuromuscular facilitation. J. Physiol. (Lond.) 195: 481-492.

Kusano, K., R. Miledi, and J. Stinnakre (1977) Acetylcholine receptors in the oocyte membrane. Nature 270: 739-741.

Kusano, K., R. Miledi, and J. Stinnakre (1982) Cholinergic and catecholaminergic receptors in the Xenopus oocyte membrane. J. Physiol. (Lond.) 328: 143-170.

Meyer, T., D. Holowka, and L. Stryer (1988) Highly cooperative opening of calcium channels by inositol 1,4,5-trisphosphate. Science 240 : 653-656.

Miledi, R. (1982) A calcium-dependent chloride current in Xenopus laevis oocytes. Proc. R. Soc. London [Biol.] 215: 491-497.

Miledi, R., and I. Parker (1984) Chloride current induced by injection of calcium into Xenopus oocytes. J. Physiol. (Lond.) 257: 173-183.

Miledi, R., and I. Parker (1989) Latencies of membrane currents evoked in Xenopus oocytes by receptor activation, inositol trisphosphate and calcium. J. Physiol. (Lond.) 405: 189-210.
Miledi, R., I. Parker, and K. Sumikawa (1987) Oscillatory chloride current evoked by temperature jumps during activation of muscarinic and serotonin receptors in Xenopus oocytes. J. Physiol. (Lond.) 383: 213-229.

Miledi, R., I. Parker, and K. Sumikawa (1989) Transplanting receptors from brains into oocytes. In Fidia Award Lecture Series, J. Smith, ed., Raven, New York.

Nerbonne, J. M. (1986) Design and application of intracellular probes. In Optical Methods in Cell Physiology, P. DeWeere and B. Salzberg, eds., pp. 418-439, Wiley, New York.

Oosawa, Y., and S. Yamagishi (1989) Rat brain glutamate receptors activate chloride channels in Xenopus oocytes coupled by inositol trisphosphate and calcium. J. Physiol. (Lond.) 408: 223-232.

Oron, Y., N. Dascal, E. Nadler, and M. Lupu (1985) Inositol 1,4,5trisphosphate mimics muscarinic response in Xenopus oocytes. Nature 313: $141-143$.

Parker, I. (1988) A threshold level of inositol trisphosphate is required to trigger intracellular calcium release in Xenopus oocytes. J. Physiol. (Lond.) 407: 98P.

Parker, I. (1989) Ionic and charge displacement currents evoked by temperature jumps in native oocytes of Xenopus laevis. Proc. R. Soc. London [Biol.] (in press).

Parker, I., and R. Miledi (1986) Changes in intracellular calcium and in membrane currents evoked by injection of inositol trisphosphate into Xenopus oocytes. Proc. R. Soc. London [Biol.] 228: 307-315.

Parker, I., and R. Miledi (1987a) Inositol trisphosphate activates a voltage-dependent calcium influx in Xenopus oocytes. Proc. R. Soc. London [Biol.] 231: 27-36.

Parker, I., and R. Miledi (1987b) Injection of inositol 1,3,4,5-tetrakisphosphate into Xenopus oocytes generates a chloride current dependent upon intracellular calcium. Proc. R. Soc. London [Biol.] 232: 59-70.

Parker, I., C. B. Gundersen, and R. Miledi (1985) Intracellular calcium-dependent and calcium-independent responses of rat brain serotonin receptors transplanted to Xenopus oocytes. Neurosci. Res. 2: $491-496$

Parker, I., K. Sumikawa, and R. Miledi (1987) Activation of a common effector system by different brain neurotransmitter receptors in Xenopus oocytes. Proc. R. Soc. London [Biol.] 231: 37-45.

Payne, R., B. Walz, S. Levy, and A. Fein (1988) The localization of calcium release by inositol trisphosphate in Limulus photoreceptors and its control by negative feedback. Phil. Trans. R. Soc. London [Biol.] 320: 359-379.

Rink, T. J., and R. Jacob (1989) Calcium oscillations in non-excitable cells. TINS $12: 43-46$.

Sladeczek, F., J.-P. Pin, M. Recasens, J. Bockaert, and S. Weiss (1985) Glutamate stimulates inositol phosphate formation in striatal neuroncs. Nature 317: 717-719.

Takahashi, T., E. Neher, and B. Sakmann (1987) Rat brain serotonin receptors in Xenopus oocytes are coupled by intracellular calcium to endogenous channels. Proc. Natl. Acad. Sci. USA 84: 5063-5067.

Walker, J. N., A. V. Somlyo, Y. E. Goldman, A. P. Somlyo, and D. R. Trentham (1987) Kinetics of smooth and skeletal muscle activation by laser pulse photolysis of caged inositol 1,4,5-trisphosphate. Nature 327: 249-251. 